\title{
Clinical characteristics of cutaneous lupus erythematosus
}

\author{
Justyna Szczęch, Maja Rutka, Dominik Samotij, Agnieszka Zalewska, Adam Reich
}

Department of Dermatology, Venereology and Allergology, Wroclaw Medical University, Wroclaw, Poland

Adv Dermatol Allergol 2016; XXXIII (1): 13-17

DOI: $10.5114 / p d i a .2014 .44031$

\begin{abstract}
Introduction: Lupus erythematosus (LE) shows a wide variety of clinical manifestations, skin involvement being one of the most important.

Aim: To analyze the clinical presentation of cutaneous variants of lupus erythematosus in terms of skin lesion spectrum and extracutaneous involvement.

Material and methods: A total of 64 patients with cutaneous LE (CLE) were included. The study was based on the "Core Set Questionnaire" developed by the European Society of Cutaneous Lupus Erythematosus (EUSCLE). Clinical severity of skin lesions was evaluated with the Cutaneous Lupus Erythematosus Disease Area and Severity Index (CLASI). All results were subjected to statistical analysis.

Results: Fifteen (23.4\%) patients had an acute CLE (ACLE), 26 (40.6\%) subacute CLE (SCLE) and 21 (32.8\%) chronic CLE (CCLE). Two (3.2\%) individuals only demonstrated urticarial vasculitis as a cutaneous manifestation of LE and these patients were excluded. Patients with ACLE were characterized by the earliest onset of the disease (mean age of 31.9 \pm 15.0 years; $p<0.001$ ). On average, $4.8 \pm 1.8$ criteria of systemic LE were found in the ACLE group compared to $2.7 \pm 1.3$ criteria in SCLE and $2.5 \pm 1.5$ criteria in CCLE $(p<0.001)$. The highest activity of skin lesions according to CLASI was found in the SCLE group $(p=0.002)$. On the other hand, the most severe skin damage was observed in CCLE $(p<0.01)$. Conclusions: Each variant of CLE differs significantly from the others in respect of various aspects of clinical manifestations. Due to a number of different variants of LE skin lesions, a unified classification of CLE still remains a challenge.
\end{abstract}

Key words: cutaneous lupus erythematosus, diagnostic criteria, CLASI, lupus erythematous, skin, Core Set Questionnaire.

\section{Introduction}

Lupus erythematosus (LE) is a chronic autoimmune disease with a wide spectrum of presenting symptoms ranging from mild cutaneous manifestations in localized cutaneous LE (CLE) to severe, life-threatening internal organ damage in systemic LE (SLE). The etiology of LE remains unknown, although the environmental, genetic, viral and hormonal factors are taken into consideration as probable causes or precipitating factors of this disease $[1,2]$.

Skin involvement is seen in about $70-85 \%$ of all LE patients [3]. Sometimes skin involvement is the only manifestation of LE, while in other cases it could rather be a mild bystander of severe internal involvement. Isolated CLE is a rare disease, albeit it is still about 2 to 3 times more frequent than SLE [4]. Cutaneous features of LE can be classified into LE-specific and LE-nonspecific ones. LEspecific skin lesions usually appear only in patients with LE and thus can be handled as diagnostic ones (e.g. "ma- lar rash", discoid lupus lesions), while LE-nonspecific skin lesions are not characteristic of LE as they can also be observed in other autoimmune processes. However, the presence of LE-nonspecific skin changes often implies systemic involvement in LE patients. The most common LE-nonspecific skin lesions are livedo reticularis and findings related to thrombophlebitis due to LE-related coagulopathy or secondary cutaneous vasculitis [5-7].

Lupus erythematosus-specific skin lesions are very heterogeneous. The most widely accepted classification of LE-specific cutaneous involvement includes acute CLE (ACLE) with its localized and generalized forms, subacute CLE (SCLE) with its annular and papulosquamous forms and chronic CLE (CCLE) including discoid LE (DLE), lupus panniculitis and chilblain lupus. Some authors postulated to separate an intermittent CLE (ICLE) group covering lupus tumidus, while others handle this subtype as a form of CCLE $[8,9]$.

Address for correspondence: Adam Reich MD, PhD, Department of Dermatology, Venereology and Allergology, Wroclaw Medical University, 1 Chałubińskiego St, 50-368 Wroclaw, Poland, phone: +48 605076 722, fax: +48 7132709 99, e-mail: adi_medicalis@go2.pl Received: 24.03.2014, accepted: 8.06.2014. 
A valid assessment of skin involvement in LE patients still remains a challenge in daily clinical settings. Several scoring systems have been proposed, but none was unanimously accepted. Lupus erythematosus skin lesions can be evaluated e.g. by Cutaneous Lupus Erythematosus Disease Area and Severity Index (CLASI), which is a very detailed and accurate measurement of CLE severity, but is also time-consuming and thus seems to be not very feasible for regular use. This scoring system has been designed to measure CLE activity and skin damage [10-12]. Severity of such features like erythema, scaling, hypertrophy, edema and infiltration in different anatomical locations are summed up to assess disease activity. Skin damage considers dyspigmentation, scarring, atrophy and panniculitis $[10,11]$. To standardize the assessment of CLE patients the European Society of Cutaneous Lupus Erythematosus developed the Core Set Questionnaire which includes six sections covering patient data, detailed diagnosis, skin involvement, CLASI, laboratory findings and treatment [13]. This questionnaire enables comparison of various CLE subtypes in a standardized and valid way.

\section{Aim}

The aim of the study was to analyze the clinical presentation of cutaneous variants of lupus erythematosus in relation to skin lesion spectrum and extracutaneous involvement.

\section{Material and methods}

A total of 64 consecutive patients ( 45 women and 19 men) diagnosed with cutaneous involvement during LE course were initially included into the study. All subjects were inpatients of the Department of Dermatology, Venereology and Allergology in Wroclaw. Their age ranged between 19 and 87 years (mean: $51.4 \pm 17.3$ years) while the age at the time of disease onset ranged between 16 and 86 years (mean: $45.9 \pm 18.8$ years). The study was conducted in accordance with the Data Protection Act and according to the ethical guidelines of the Declaration of Helsinki. The study was approved by our institutional review board. All patients agreed to participate in the study. Among analyzed subjects, 15 (23.4\%) patients were diagnosed as having an acute CLE (ACLE) (8 patients with localized and 7 with generalized form), $26(40.6 \%)$ as having subacute CLE (SCLE) (including 17 patients with annular and 9 with papulosquamous form), and 21 (32.8\%) as having chronic CLE (CCLE) - all with discoid LE. Two (3.2\%) individuals only demonstrated urticarial vasculitis as a cutaneous manifestation of LE and these patients were excluded from further analysis as urticarial vasculitis is nowadays considered as a LE-nonspecific skin manifestation. The disease was confirmed by histological examination in 41 (66.1\%) in- dividuals, while remaining patients (33.9\%) had the disease diagnosed solely based on clinical presentation and laboratory abnormalities.

The study was performed between 2007 and 2012. It was based on the "Core Set Questionnaire" of the European Society of Cutaneous Lupus Erythematosus (EUSCLE), which includes data gained during a detailed anamnesis, physical examination and certain laboratory tests [13]. The EUSCLE Core Set Questionnaire was approved by the central Ethical Committee of the University of Muenster in Germany. The study was performed using 1997 update of the 1982 American College of Rheumatology Revised Criteria for Classification of Systemic Lupus Erythematosus [14, 15]. Diagnosis of CLE was based on clinical presentation, histopathology, direct immunofluorescence (lupus band test) and additional laboratory data, if necessary $[16,17]$. Severity of skin lesions was evaluated according to the Cutaneous Lupus Erythematosus Disease Area and Severity Index (CLASI) [10]. Antinuclear antibodies (ANA) were detected by immunofluorescence on HEp2 cells and immunoblot tests. A titer above 1 : 160 was considered as a positive test result. If indicated, the lupus band test was performed from lesional and/or non-lesional skin.

\section{Statistical analysis}

All results were analysed statistically using the software package Statistica ${ }^{\circledR} 10.0$ (Statsoft, Krakow, Poland). The significance of the observed differences between studied groups was determined by analysis of variance (ANOVA), Student $t$ test, and $\chi^{2}$ test or two sided Fisher exact test, where appropriate. A $p$-value lower than 0.05 was considered as statistically significant.

\section{Results}

\section{Demographic data}

Patients with ACLE were characterized by the earliest onset of the disease (mean age: $31.9 \pm 15.0$ years), followed by CCLE and SCLE (mean age: $43.9 \pm 14.2$ years and $55.6 \pm 18.9$ years, respectively; $p<0.001$ ). Earlier disease onset also resulted in the younger age of ACLE patients (mean age: $39.0 \pm 15.5$ years) compared to CLE and SCLE individuals ( $49.1 \pm 12.8$ years and $60.5 \pm 16.8$ years, respectively, $p<0.001)$. Female predominance was higher in ACLE group (females : males ratio $-4: 1$ ) than in SCLE $(2.2: 1)$ and CCLE groups $(1.6: 1)$, but the difference was not significant $(p=0.51)$. The highest prevalence of cigarette smoking was observed in CCLE patients (85.7\%); followed by SCLE $-57.7 \%$, and ACLE - 53.3\% $(p<0.05)$.

\section{Severity of cutaneous involvement}

According to CLASI, the highest activity of skin lesions was found in SCLE group (mean: $26.4 \pm 14.5$ points, range: 4-58 points) followed by ACLE (mean: $16.7 \pm 8.3$ points, 
range: $5-34$ points) and CCLE subjects (mean: $12.9 \pm 11.9$ points, range: $3-55$ points) $(p=0.001)$. On the other hand, the most severe skin damage was observed in CCLE (mean: $5.0 \pm 4.9$ points, range: $0-18$ points) followed by ACLE (mean: $2.5 \pm 3$,0 points, range: $0-9$ points) and SCLE (mean: $1.8 \pm 2.1$ points, range: $0-8$ points) $(p<0.01)$.

\section{Systemic involvement in CLE patients}

Systemic symptoms were present in 14 (93.3\%) patients with ACLE, 15 (57.7\%) with SCLE and 6 (28.6\%) with CCLE ( $p<0.001)$. At least 4 out of 11 ACR (American College of Rheumatology) diagnostic criteria indicating the diagnosis of SLE were observed in 12 (80\%) subjects with ACLE (mean: $5.1 \pm 1.7$ criteria, range: $3-9$ criteria), 7 (26.9\%) with SCLE (mean: $3.0 \pm 1.3$ criteria, range: $0-5$ criteria) and 7 (33.3\%) with CCLE (mean: $2.7 \pm 1.5$ criteria, range: $0-7$ criteria) $(p<0.01)$.

Arthritis, neurological involvement and oral ulcers more commonly accompanied ACLE (60\%, 33.3\% and $33.3 \%$ of patients, respectively), compared to SCLE (19.2\%, $11.5 \%$ and $7.7 \%$, respectively) and CCLE (19.0\%, $4.8 \%$ and $4.8 \%$, respectively) ( $p=0.01, p<0.05$ and $p=0.02$, respectively). Serositis was only observed in
$2(13.2 \%)$ patients with ACLE and 1 (3.8\%) with SCLE $(p=0.18)$, renal disease in $3(20.0 \%)$ with ACLE, 1 (3.8\%) with SCLE and 1 (4.8\%) with CCLE ( $p=0.15)$. Hematologic abnormalities were nearly equally frequent in ACLE and SCLE (53.3\% and 42.3\%, respectively), but were uncommon in CCLE individuals $(14.3 \%, p<0.05)$ (Table 1$)$.

The secondary Sjögren's syndrome more often occurred in the SCLE group (23.1\%) than in ACLE (13.3\%). Interestingly, none of CCLE patients demonstrated symptoms of sicca syndrome $(p=0.06)$. The prevalence of photosensitivity was high in all groups, with the highest prevalence in the ACLE group (93.3\%), followed by SCLE (76.9\%) and CCLE (71.4\%) ( $p=0.27)$.

\section{Immunological disturbances in CLE patients}

Presence of ANA was confirmed for $86.7 \%$ of all patients with ACLE and for $78.6 \%$ of patients with SCLE. Regarding ANA subtypes, anti-La (33.3\%), anti-Sm (33.3\%) anti-RNP (26.7\%) and anti-dsDNA (33.3\%) antibodies were the most frequent in the ACLE group, while anti-Ro (50.0\%) antibodies were most commonly found in SCLE subjects (Table 2). Regarding ACR diagnostic criteria for SLE, presence of anti-Sm and anti-RNP antibodies were

Table 1. Frequency of American College of Rheumatology diagnostic criteria for systemic lupus erythematosus in different subtypes of cutaneous lupus erythematosus

\begin{tabular}{|c|c|c|c|c|}
\hline Variable & ACLE (\%) & SCLE (\%) & CCLE (\%) & Value of $p$ \\
\hline Malar rash & 86.7 & 30.8 & 23.8 & $<0.001$ \\
\hline Discoid rash & 20.0 & 11.5 & 90.5 & $<0.001$ \\
\hline Photosensitivity & 93.3 & 76.9 & 71.4 & 0.02 \\
\hline Oral ulcers & 33.3 & 7.7 & 4.8 & 0.02 \\
\hline Arthritis & 60.0 & 19.2 & 19.0 & 0.01 \\
\hline Serositis & 13.2 & 3.8 & 0.0 & 0.18 \\
\hline Renal disorder & 20.0 & 3.8 & 4.8 & 0.15 \\
\hline Neurological disorder & 33.3 & 11.5 & 4.8 & $<0.05$ \\
\hline Hematologic disorder & 53.3 & 42.3 & 14.3 & 0.03 \\
\hline Immunological disorder & 13.3 & 15.4 & 0.0 & 0.19 \\
\hline Antinuclear antibodies & 86.7 & 80.9 & 42.9 & $<0.01$ \\
\hline
\end{tabular}

ACLE - acute cutaneous lupus erythematosus, SCLE - subacute cutaneous lupus erythematosus, CCLE - chronic cutaneous lupus erythematosus; $p$-values according to $\chi^{2}$ test.

Table 2. Presence of various antinuclear antibodies in different subtypes of cutaneous lupus erythematosus

\begin{tabular}{lcccc}
\hline Type of antibody & ACLE (\%) & SCLE (\%) & CCLE (\%) & Value of $p$ \\
\hline Anti-Ro & 46.7 & 50.0 & 4.8 & 0.002 \\
\hline Anti-La & 33.3 & 26.9 & 4.8 & 0.07 \\
\hline Anti-Sm & 33.3 & 0.0 & 0.0 & $<0.001$ \\
\hline Anti-RNP & 26.7 & 0.0 & 4.8 & $<0.02$ \\
\hline Anti-histone & 0.0 & 11.5 & 0.0 & 0.24 \\
\hline Anti-dsDNA & 33.3 & 15.4 & 4.8 & 0.07 \\
\hline
\end{tabular}

ACLE - acute cutaneous lupus erythematosus, SCLE - subacute cutaneous lupus erythematosus, CCLE - chronic cutaneous lupus erythematosus; $p$-values according to $\chi^{2}$ test. 
significantly associated with hematological disturbances (100\% vs. $25.9 \%$; $p<0.01$ and $100 \%$ vs. $28.6 \%$; $p<0.01$, respectively). Anti-Ro antibodies were also significantly related to hematological disturbances $(72.7 \%$ vs. $19.0 \%$; $p<0.01)$ as well as to erosions in the oral cavity $(36.4 \%$ vs. $4.8 \% ; p=0.02)$, anti-La antibodies to arthritis $(80.0 \%$ vs. $29.6 \%, p<0.05)$ and hematological disturbances (80.0\% vs. 29.6\%; $p<0.05)$, while anti-dsDNA antibodies to malar rash ( $100 \%$ vs. $40.7 \% ; p=0.01)$. A decreased level of complement components (C3, C4) in serum was observed in $45.5 \%$ of patients with ACLE and in $43.8 \%$ of SCLE individuals, whereas all patients with CCLE had a normal complement level $(p<0.05)$.

\section{Discussion}

The vast majority of LE patients demonstrate some skin abnormalities, which usually are divided into LE-specific and LE-unspecific lesions [18]. Based on LE-specific skin manifestations, various clinical subtypes of CLE have been differentiated, however, the clinical classification of CLE still remains a matter of debate. For instance, there is still no agreement if lupus erythematosus tumidus should be considered as a subtype of CCLE or has to be differentiated into a separate category of intermittent CLE (ICLE) [17, 18]. In addition, some LE-unspecific skin lesions, like urticarial vasculitis or erythema multiformelike LE lesions, might be quite specific and can represent a distinct CLE subtype. Furthermore, it is sometimes observed that one patient may demonstrate more than one variant of LE-specific skin lesions, a phenomenon which further complicates CLE classification.

We do believe that in order to have a better insight into CLE pathology and to be able to develop a more suitable classification of CLE, we should undertake any effort to collect more data on CLE patients from different centers and different patient populations. To standardize data on CLE subjects collected from different European countries, EUSCLE has recently developed a structured questionnaire, which, in our opinion, might be very helpful in conducting cross-sectional, population-based studies on CLE subjects [13]. Our study provides further supporting data that the EUSCLE "Core Set Questionnaire" seems to be a valuable tool for a standardized clinical assessment of CLE patients which might be useful in conducting future studies on different patient populations.

Another important clinical issue of CLE evaluation is the assessment of the severity of skin lesions. In 2005, Albrecht et al. [10] validated the CLASI - a new measurement instrument for CLE that can be used in clinical trials. It was demonstrated that the activity scoring system of CLASI very well correlated with the general assessment of the global skin health performed by physicians and by patients [19]. Our study also showed that CLASI is a valid method of CLE assessment, which also enables differentiation of different CLE subtypes. However, this scale is time consuming and requires some clinical expertise to be properly scored, and thus is not well suited for daily clinical practice. Furthermore, it is still unknown if it is feasible for rare variants of CLE, like LE panniculitis or chilblain LE. Some modifications of CLASI have been proposed, but to date no instrument assessing CLE severity really overcame previous shortcomings [20, 21]. Furthermore, to the best of our knowledge, no currently available CLE severity scoring system takes into consideration subjective symptoms and patient's perspective of having skin lesions in LE. Remarkably, it has recently also been shown that damage domain of CLASI did not correlate with overall health-related quality of life of CLE patients [22].

In the current study we have observed that systemic symptoms of LE may be found in all analyzed CLE subtypes, albeit with different prevalence. We have found this observation important, as some subtypes of CLE, like e.g. SCLE, were not included in the original ACR diagnostic criteria of SLE, which might lead to underrecognition of SLE $[14,15]$. It might also cause a misconception that SCLE is a CLE variant without systemic involvement. On the other hand, photosensitivity, i.e. "unusual reaction to sunlight by a patient's history or by physician observation", was considered as a separate diagnostic criterion of SLE $[14,15]$, which might lead to a faulty increase in the number of positive SLE criteria in selected patients, as "malar rash" is often indistinguishable from photosensitivity, and therefore these criteria were not independent [23]. These shortcomings have been substantially improved in the new diagnostic criteria proposed by Petri et al. [24], as they recognized many previously excluded cutaneous LE manifestations, including SCLE and LE tumidus.

It must be underlined that the current study was performed on patients treated in the dermatology department, which might differ from LE subjects treated by rheumatologists regarding the distribution pattern of cutaneous manifestations. Having only patients from dermatology department might be considered as a limitation of our study. Thus, it would be interesting to perform a similar study on LE individuals from the rheumatology department to test whether these patients indeed have different skin problems than those from dermatology.

Lupus erythematosus is often accompanied by ANA directed against various nuclear antigens. These antibodies have crucial diagnostic and prognostic meaning, however, no ANA subtype could be solely linked with one clinical subtype of CLE, albeit some of them may be more common in certain CLE variants. Interestingly, it seems that some ANA subtypes may predispose patients to particular systemic manifestations of LE, e.g. antibodies anti-Ro were found to be related to a higher prevalence of oral ulcers and hematological disturbances. 


\section{Conclusions}

It can be concluded that each variant of CLE differs significantly from the others in respect of various aspects of clinical manifestations. Due to a number of different variants of LE skin manifestations, a unified classification of CLE still remains a challenge. We do believe that EUSCLE Core Set Questionnaire might be helpful in a standardized assessment of clinical characteristics of different CLE subtypes. However, future attempts should be concentrated on the development of better instruments for CLE severity assessment, which will also consider patient perspective on skin lesions and will be simple enough to be used in daily clinical practice.

\section{Conflict of interest}

The authors declare no conflict of interest.

\section{References}

1. Jasiuk B, Reich A. The role of apoptosis in the pathogenesis of systemic lupus erythematosus. Dermatol Klin 2005; 7: 97-100.

2. Shabgah AG, Fattahi E, Shahneh FZ. Interleukin-17 in human inflammatory diseases. Adv Dermatol Allergol 2014; 31: 256-61.

3. Vera-Recabarren MA, Garcia-Carrasco M, Ramos-Casals M, Herrero C. Cutaneous lupus erythematosus: clinical and immunological study of 308 patients stratified by gender. Clin Exp Dermatol 2010; 35: 729-35.

4. Tebbe B, Orfanos CE. Epidemiology and socioeconomic impact of skin disease in lupus erythematosus. Lupus 1997; 6: 96-104

5. Cardinali C, Caproni M, Bernacchi E, et al. The spectrum of cutaneous manifestations in lupus erythematosus - the Italian experience. Lupus 2000; 9: 417-23.

6. D'Cruz D. Vasculitis in systemic lupus erythematosus. Lupus 1998; 7: 270-4.

7. Fabbri P, Cardinali C, Giomi B, Caproni M. Cutaneous lupus erythematosus: diagnosis and management. Am J Clin Dermatol 2003; 4: 449-65.

8. Parodi A, Caproni M, Cardinali C, et al. Clinical, histological and immunopathological features of 58 patients with subacute cutaneous lupus erythematosus. Dermatology 2000; 200: 6-10.

9. Schmitt V, Meuth AM, Amler S, et al. Lupus erythematosus tumidus is a separate subtype of cutaneous lupus erythematosus. Br J Dermatol 2010; 162: 64-73.

10. Albrecht J, Taylor L, Berlin JA, et al. The CLASI (Cutaneous Lupus Erythematosus Disease Area and Severity Index): an outcome instrument for cutaneous lupus erythematosus. J Invest Dermatol 2005; 125: 889-94.

11. Bein D, Kuehn E, Meuth AM, et al. Evaluation of disease activity and damage in different subtypes of cutaneous lupus erythematosus using the CLASI. J Eur Acad Dermatol Venerol 2011; 25: 652-9.

12. Bednarek A, Bartoszak L, Samborski W. Case report on a patient with lupus panniculitis. Adv Dermatol Allergol 2015; 22: 59-62.

13. Kuhn A, Patsinakidis N, Bonsmann G. The impact of the EUSCLE Core Set Questionnaire for the assessment of cutaneous lupus erythematosus. Lupus 2010; 19: 1144-52.
14. Tan EM, Cohen AS, Fries JF, et al. The 1982 revised criteria for the classification of systemic lupus erythematosus. Arthritis Rheum 1982; 25: 1271-7.

15. Hochberg MC. Updating the American College of Rheumatology revised criteria for the classification of systemic lupus erythematosus. Arthritis Rheum 1997; 40: 1725.

16. Kuhn A, Gensch K, Ständer S, Bonsmann G. Cutaneous lupus erythematosus. Part 1: clinical manifestations and classification. Hautarzt 2006; 57: 251-67.

17. Kuhn A, Ruzicka T. Classification of cutaneous lupus erythematosus. In: Cutaneous lupus erythematosus. Kuhn A, Lehmann P, Ruzicka T (eds). Springer, Heidelberg 2004; 53-8.

18. Walling HW, Sontheimer RD. Cutaneous lupus erythematosus: issues in diagnosis and treatment. Am J Clin Dermatol 2009; 10: 365-81.

19. Bonilla-Martinez ZL, Albrecht J, Troxel AB, et al. The cutaneous lupus erythematosus disease area and severity index: a responsive instrument to measure activity and damage in patients with cutaneous lupus erythematosus. Arch Dermatol 2008; 144: 173-80.

20. Kuhn A, Meuth AM, Bein D, et al. Revised Cutaneous Lupus Erythematosus Disease Area and Severity Index (RCLASI): a modified outcome instrument for cutaneous lupus erythematosus. Br J Dermatol 2010; 163: 83-92.

21. Kreuter A. A revised scoring system for cutaneous lupus erythematosus: the RCLASI. Br J Dermatol 2010; 163: 3.

22. Verma SM, Okawa J, Propert KJ, Werth VP. The impact of skin damage due to cutaneous lupus on quality of life. $\mathrm{Br}$ J Dermatol 2014; 170: 315-21.

23. Albrecht J, Berlin JA, Braverman IM, et al. Dermatology position paper on the revision of the 1982 ACR criteria for systemic lupus erythematosus. Lupus 2004; 13: 839-49.

24. Petri M, Orbai AM, Alarcón GS, et al. Derivation and validation of the Systemic Lupus International Collaborating Clinics classification criteria for systemic lupus erythematosus. Arthritis Rheum 2012; 64: 2677-86. 